\title{
Blood supply characteristics of pedunculated hepatocellular carcinoma prior to and following transcatheter arterial chemoembolization treatment: An angiographic demonstration
}

\author{
DEXIAO HUANG ${ }^{1,2}$, YONG CHEN ${ }^{2}$, QINGLE ZENG ${ }^{2}$, JIANBO ZHAO ${ }^{2}$, \\ XIZHONG WU ${ }^{1}$, RENHUA WU ${ }^{1}$ and YANHAO $\mathrm{LI}^{2}$ \\ ${ }^{1}$ Department of Medical Imaging, The Second Affiliated Hospital, Medical College of Shantou University, \\ Shantou, Guangdong 515041; ${ }^{2}$ Department of Interventional Radiology, Nanfang Hospital, \\ Southern Medical University, Guangzhou, Guangdong 510515, P.R. China
}

Received August 2, 2016; Accepted November 29, 2017

DOI: $10.3892 / \mathrm{ol} .2018 .7844$

\begin{abstract}
Pedunculated hepatocellular carcinoma (P-HCC) is a rare type of $\mathrm{HCC}$, defined as a carcinoma protruding from the liver with or without a pedicle with a low degree of liver invasion. The present study aimed to evaluate the characteristics of blood supply of P-HCC prior to and following transcatheter arterial chemoembolization (TACE) treatment. Angiographic findings prior to and following TACE treatment in 39 patients with P-HCC were analyzed retrospectively. Angiography performed at the first TACE session revealed 70 tumor-feeding arteries collectively in all patients, including $31 / 70(44.0 \%)$ extrahepatic parasitic arteries in $23 / 39$ patients (59.0\%). The intrahepatic arteries served as the main blood supply to P-HCC in all patients. Extrahepatic collateral blood supplies to $\mathrm{P}-\mathrm{HCC}$ s were significantly associated with larger tumor diameter $\left(\chi^{2}=164.000, \mathrm{P}<0.001\right)$, but not tumor location $\left(\chi^{2}=7.358, \mathrm{P}=0.061\right)$. Following repeated TACE treatment, all angiographies revealed a total of 131 tumor feeding arteries collectively in all patients, including intrahepatic arteries (54/131) and extrahepatic collateral arteries (78/131) in 31 patients $(79.5 \%)$. Compared with angiographies performed at the initial TACE treatment, these results also demonstrated
\end{abstract}

Correspondence to: Professor Renhua Wu, Department of Medical Imaging, The Second Affiliated Hospital, Medical College of Shantou University, 69 Dongxia North Road, Shantou, Guangdong 515041, P.R. China

E-mail: rhwu@stu.edu.cn

Professor Yong Chen, Department of Interventional Radiology, Nanfang Hospital, Southern Medical University, 1838 Guangzhou Avenue North, Guangzhou, Guangdong 510515, P.R. China E-mail: cheny102@163.com

Key words: pedunculated hepatocellular carcinoma, transcatheter arterial chemoembolization, angiography, extrahepatic collateral supply an increase in the number of extrahepatic collateral arteries, which produced 47 new blood vessels $\left(\chi^{2}=4.278, \mathrm{P}=0.039\right)$. $\mathrm{P}-\mathrm{HCC}$ tumor lesions readily acquired a parasitic blood supply from adjacent vessels following repeated TACE. Intrahepatic arteries functioned as the main blood supply for P-HCC, whereas extrahepatic collateral arteries were complementary to P-HCC, regardless of whether the patient was pre- or post-TACE. Extrahepatic collateral supplies to P-HCCs that originated from adjacent vessels were rich, were closely associated with tumor size, and were prone to be newly established following repeated TACE.

\section{Introduction}

Pedunculated hepatocellular carcinoma (P-HCC) is a rare type of HCC, which is defined as a carcinoma protruding from the liver with or without a pedicle, and with a low degree of liver invasion (1). P-HCC was first described by Roux in 1897 (2), and represents $0.24-3.00 \%$ of all cases of HCC in Japan $(3,4)$. Additionally, to the best of our knowledge, $<200$ cases have been reported in previous studies (4-6).

The majority of P-HCC cases are treated surgically, with higher operability rates and better survival than conventional HCC, which may be due to its unique growth pattern, high rate of tumor capsule formation and lower vascular invasion (4-6). However, up to $39.4 \%$ of patients with $\mathrm{P}-\mathrm{HCC}$ are unable to undergo surgical resection (7). Therefore, palliative treatments may serve a central function in the treatment of unresectable $\mathrm{P}-\mathrm{HCC}$. Transcatheter arterial chemoembolization (TACE) is the most widely used locoregional therapy for patients with intermediate HCC, who cannot be treated surgically (8-11). However, the blood supply to P-HCC is complicated, arising from hepatic arteries and extrahepatic collateral vessels (3). $\mathrm{P}-\mathrm{HCC}$ tumor lesions protruding from the liver may receive an extrahepatic blood supply from adjacent vessels, despite the presence of a patent hepatic artery. In addition, repeated TACE results in the attenuation of hepatic arterial circulation and causes the development of extrahepatic collaterals (12). Therefore, the characteristics of the blood supply of P-HCC may aid the improvement of the therapeutic effect of transcatheter 
management and determine the selection of subsequent treatment schemes for patients with P-HCC following TACE.

The aim of the present study was to retrospectively analyze angiographic findings in 39 patients with P-HCC treated with TACE, and to evaluate the blood supply characteristics of $\mathrm{P}-\mathrm{HCC}$ prior to and following TACE treatment.

\section{Patients and methods}

Patients. Between January 2003 and February 2016, 39 patients (male, 37; female, 2; mean age, 49 years; age range, 19 to 70 years) with histologically proven P-HCC, out of a total of 1,238 patients with HCC, were treated in the Department of Interventional Radiology, from the Nanfang Hospital of Southern Medical University and The Second Affiliated Hospital of Shantou University Medical College (Guangdong, China). Exclusion criteria were as follows: i) Karnofsky performance score of $<80$; ii) hepatic function analyzed using the Child-Pugh classification C (8); iii) vascular tumor thrombus; iv) extrahepatic metastases (not including regional lymph node involvement); v) received previous treatment for this type of tumor. P-HCC was defined, using computed tomography (CT) scanning or magnetic resonance imaging (MRI), as HCC with tumor lesions protruding from the liver with or without a pedicle. A total of 39 patients with $\mathrm{P}-\mathrm{HCC}$ were treated with TACE (between 2 and 9 sessions) in the present study. The P-HCC tumor lesions ranged between 4.2 and $22.1 \mathrm{~cm}$ (mean, $10.2 \mathrm{~cm}$ ) in diameter, including $<5 \mathrm{~cm}$, $\mathrm{n}=2 ; 5-10 \mathrm{~cm}, \mathrm{n}=19$; and $>10 \mathrm{~cm}, \mathrm{n}=18$, and were localized within the right diaphragmatic surface $(n=2)$, the right visceral surface $(n=20)$, the left diaphragmatic surface $(n=2)$ and the left visceral surface $(n=15)$ (Table I).

CT scanning or MRI examination and laboratory tests, including quantification of routine blood test, liver function, coagulation function and $\alpha$-fetoprotein (AFP) levels, were regularly performed prior to angiography. Written informed consent was obtained from all patients prior to treatment.

Angiography. Angiographies were performed using the Seldinger technique (13). Subsequent to introducing a 4 or 5-F catheter through the femoral artery, arteriograms of the celiac, common hepatic and superior mesenteric arteries were initially performed in all patients to localize lesions and identify blood vessels feeding the tumor. During selected catheterization, the catheter must be placed at the arterial orifice; however, it cannot be entered too deeply into the arteries orifice as this may omit extrahepatic collateral supplies. A microcatheter was used for highly selective catheterization when the tumor-feeding vessel was small and twisted. Extrahepatic collateral pathways were sought when a tumor stain not corresponding to P-HCC, as depicted by imaging modalities including contrast-enhanced CT and MRI, was not identified on angiograms of these arteries. $\mathrm{CT}$ and MRI results obtained prior to the TACE procedure, which can depict the tumor and guide the TACE procedure. The individual vessels, which may feed HCC, depending on the tumor location, were selected to determine whether collateral supply to the tumor was present. Aortography aided the location of the individual vessels arising from the aorta, if required.
Statistical analysis. All data were presented as the percentage of patients or the mean \pm standard deviation. Data were compared using Pearson's $\chi^{2}$ tests when appropriate. $\mathrm{P}<0.05$ was considered to indicate a statistically significant difference. All statistical analyses were performed using SPSS software (version 17.0; SPSS, Inc., Chicago, IL, USA).

\section{Results}

Angiographic results prior to TACE. Angiographies at the first TACE session revealed 70 tumor-feeding arteries in the 39 patients, including $39(56.0 \%)$ intrahepatic arteries and 31 $(44.0 \%)$ extrahepatic collateral vessels in 23 cases $(23 / 39)$, which consisted of 14 cases with a tumor $>10 \mathrm{~cm}$ in size $(5 / 14$ had two extrahepatic collateral arteries) and 9 cases with a tumor $5-10 \mathrm{~cm}$ in size (3/9 had two extrahepatic collateral arteries) (Figs. 1-4). All intrahepatic arteries served as the main blood supply for the P-HCC in all patients. Extrahepatic collateral vessels originated from the gastroduodenal arteries (11/31), left gastric arteries (6/31), superior mesenteric arteries (5/31), pancreaticoduodenal arteries $(4 / 31)$, inferior phrenic artery (3/31) and right adrenal arteries (2/31) (Table II). Extrahepatic collateral blood supply to the P-HCC were significantly associated with a larger tumor size $\left(\chi^{2}=164.000, \mathrm{P}<0.001\right)$; however, no significant association was identified with regards to the tumor location $\left(\chi^{2}=7.358\right.$, $\mathrm{P}=0.061$ ) (Table I), which demonstrated that the larger the $\mathrm{P}-\mathrm{HCC}$ tumor size, the greater the number of extrahepatic collateral supplies.

Angiographic results subsequent to repeated TACE. Following repeated TACE (2-9 sessions), angiography revealed a total of 131 tumor-feeding arteries in all patients, with 54 (41.2\%) intrahepatic arteries adding new intrahepatic collateral vessels (14/54) (Fig. 3), and 78 (58.8\%) extrahepatic collateral vessels from 31 cases $(79.5 \%)$; these vessels arose from gastroduodenal arteries (23/78), left gastric arteries (13/78), inferior phrenic artery (11/78), superior mesenteric arteries (10/78), pancreaticoduodenal arteries (8/78), right adrenal arteries (8/78), right gastric arteries (2/78), lumbar arteries (2/78) and the intercostal artery (1/78). Compared with angiographies at the initial TACE, 47 new extrahepatic blood vessels were added. These results reveal that the number of extrahepatic collateral vessels significantly increased following TACE $\left(\chi^{2}=4.278\right.$, $\mathrm{P}=0.039$; Table II).

All angiographies revealed that the intrahepatic arteries served as the main blood supply for P-HCC, whereas the extrahepatic collateral arteries served complementary functions in P-HCC, regardless of whether the patient was pre- or post-TACE (Figs. 1-4). Additionally, P-HCC tumor lesions were prone to acquire parasitic blood supplies from adjacent vessels following repeated TACE (Figs. 2 and 5).

\section{Discussion}

P-HCC is characterized by a rich blood supply, similar to HCC. All angiographies conducted in the present study revealed that the intrahepatic arteries function as the main blood supply, with the extrahepatic collateral arteries supplying a complementary blood supply for P-HCC, regardless of whether the patient is 
Table I. Blood supply, tumor size, and tumor location in 39 patients with pedunculated hepatocellular carcinoma prior to transcatheter arterial chemoembolization.

\begin{tabular}{|c|c|c|c|c|}
\hline Parameter & $\begin{array}{l}\text { Only from intrahepatic } \\
\text { arteries }^{\mathrm{a}}, \mathrm{n}\end{array}$ & $\begin{array}{l}\text { Coupling with extrahepatic } \\
\text { collateral arteries }^{b}, \mathrm{n}\end{array}$ & $\chi^{2}$ & P-value \\
\hline Tumor size, $\mathrm{cm}$ & & & 164.000 & $<0.001$ \\
\hline$<5$ & 2 & 0 & & \\
\hline $5-10$ & 19 & 5 & & \\
\hline$>10$ & 18 & 18 & & \\
\hline Tumor location & & & 7.358 & 0.061 \\
\hline Right diaphragmatic surface & 2 & 0 & & \\
\hline Right visceral surface & 20 & 13 & & \\
\hline Left diaphragmatic surface & 2 & 0 & & \\
\hline Left visceral surface & 15 & 10 & & \\
\hline
\end{tabular}

${ }^{\mathrm{a}} \mathrm{n}=39 .{ }^{\mathrm{b}} \mathrm{n}=23$.
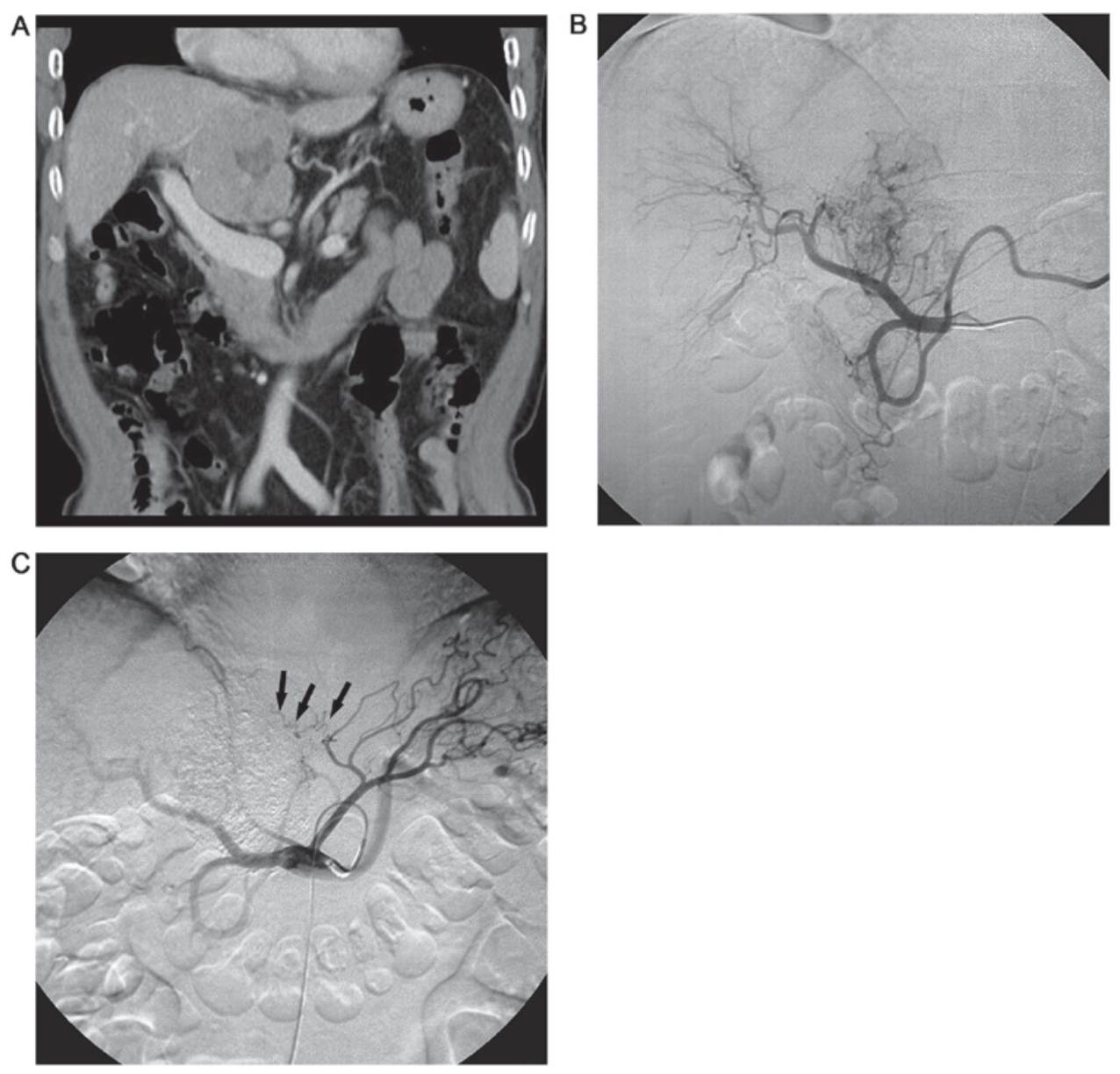

Figure 1. Angiography of a 58-year-old man with P-HCC. (A) Tumor protruding from the caudate lobe of the liver. (B) A celiac arteriogram reveals a tumor stain mainly supplied blood by the left hepatic artery, but the tumor stain corresponding to P-HCC, depicted on contrast-enhanced computed tomography, was not observed in angiograms of these arteries. (C) Extrahepatic collateral supply is identified and originates from the left gastric artery (arrows). The catheter is slightly withdrawn and placed at arterial orifice of the celiac. P-HCC, pedunculated hepatocellular carcinoma.

pre- or post-TACE. Extrahepatic collateral supplies to P-HCC are rich, and are significantly associated with tumor size; these blood supplies often arise from the feeding arteries of the organs adjoining tumor lesion and are readily established following repeated TACE (14). Extrahepatic collateral supplies to P-HCC are established under various conditions (15-17). These collateral 

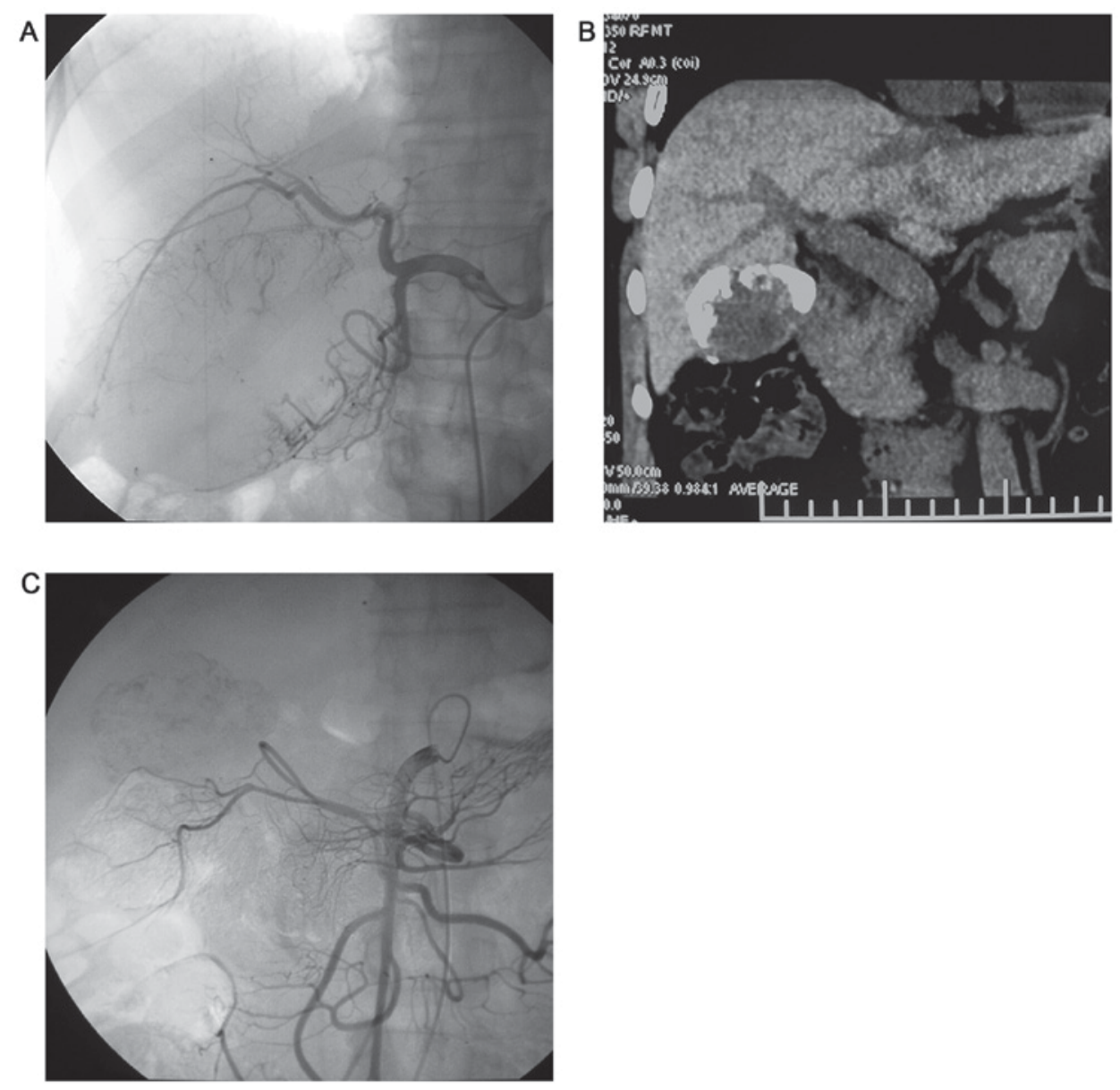

Figure 2. Images from a 47-year-old man with an extrahepatic tumor growth on the right visceral surface. (A) Angiography at the first TACE session revealed that the tumor received blood from the right hepatic artery and gastroduodenal artery. (B) Follow-up CT image obtained 1 month after the second TACE, revealing poor lipiodol deposition in the tumor lesion. (C) Angiography at the third TACE reveals that the right hepatic artery functioned as the main blood supply, and a new extrahepatic collateral supply to pedunculated hepatocellular carcinoma was arising from a branch of superior mesenteric artery. TACE, transcatheter arterial chemoembolization.

supplies may develop following the interruption of the hepatic artery by surgical ligation, arterial injury induced by repeated TACE or the placement of a catheter (15-17). Adhesions between tumors protruding from the liver and adjacent organs can exaggerate the degree of extrahepatic collateral blood supplies, although the hepatic arterial supply remains intact $(3,15,18-22)$. In the present study, extrahepatic collateral blood supplies to P-HCC commonly arose from gastroduodenal arteries, left gastric arteries, phrenic arteries, superior mesenteric arteries, pancreaticoduodenal arteries and right adrenal arteries. In patients with P-HCC, various extrahepatic collateral vessels develop and supply the tumor (3,18-29). Compared with those observed via angiographies at the initial TACE, up to $79.5 \%$ of the patients in the present study had extrahepatic collateral supplies following subsequent TACE; the results also revealed an increasing trend in the number of extrahepatic collateral vessels as the number of TACE treatments increased. Therefore, it was hypothesized that the main cause of the development of extrahepatic collaterals was attenuation of the hepatic arterial circulation by TACE (18-28).

Technically, angiographies of blood supplies to the liver, including the celiac, common hepatic and superior mesenteric arteries, should be initially performed during TACE in all patients with $\mathrm{P}-\mathrm{HCC}$, as the intrahepatic arteries manifest as the main blood supply to P-HCC (14). An arteriogram of the inferior phrenic artery, which is a major source of diaphragmatic blood supply to the liver $(12,19,22-26,29)$, is routinely performed in patients who have an interrupted hepatic arterial circulation owing to previous treatment, or in patients with tumors located near the diaphragm, which may be identified in the initial angiography.

Extrahepatic collateral blood supplies are sought when a tumor stain that corresponds to P-HCC, identified using imaging modalities in terms of the location and size of the tumor, is not present on the angiograms of these arteries. Extrahepatic collateral blood supplies are obtained through nearby blood vessels attributed to P-HCC protruding from the liver, exaggerating the degree of extrahepatic collateral blood supply sourced from adjacent organs (18-22). In practice, the catheter must be placed at the arterial orifice to avoid omitting the origin of the extrahepatic collateral blood supply during the selective catheterization. Use of a microcatheter is required for highly selective catheterization when tumor-feeding branches that arise from the extrahepatic collaterals are difficult to catheterize, owing to their branching phenotype (23). These branches are usually of small caliber and branch at acute angles, giving a twisted appearance.

$\mathrm{P}-\mathrm{HCC}$ is primarily treated by surgical resection, as a wider resection margin may be obtained, and patients have a higher 

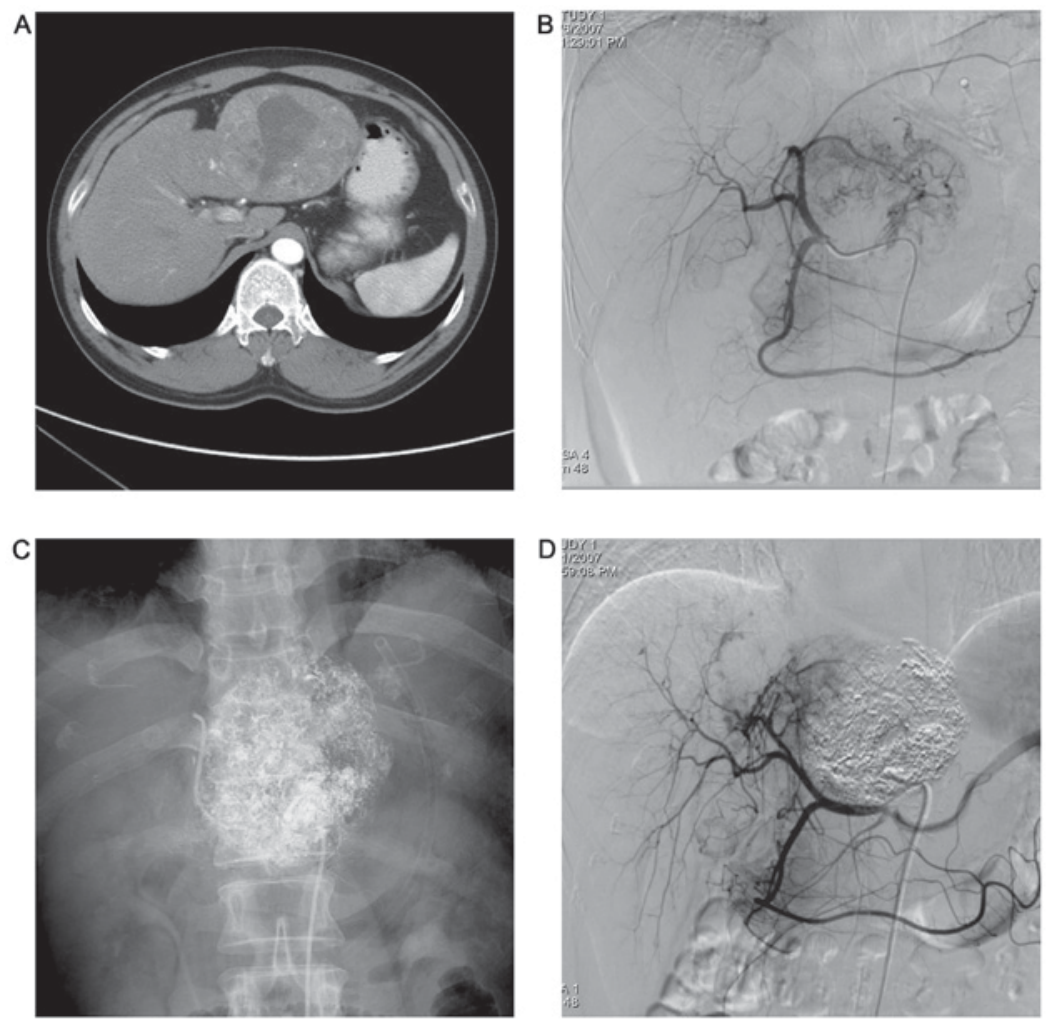

Figure 3. Angiography of a 46-year-old man with P-HCC. (A) Extrahepatic tumor growth on the left visceral surface. (B) A hepatic arteriogram reveals that the left hepatic artery is the tumor-feeding artery. (C) The catheter is advanced into the left hepatic artery, followed by arterial embolization. The radiograph obtained immediately following TACE reveals a solid chemotherapeutic agents lipiodol emulsion deposition. (D) Angiography at the third TACE revealing a new intrahepatic collateral vessel supplying blood to the P-HCC. P-HCC, pedunculated hepatocellular carcinoma; TACE, transcatheter arterial chemoembolization.
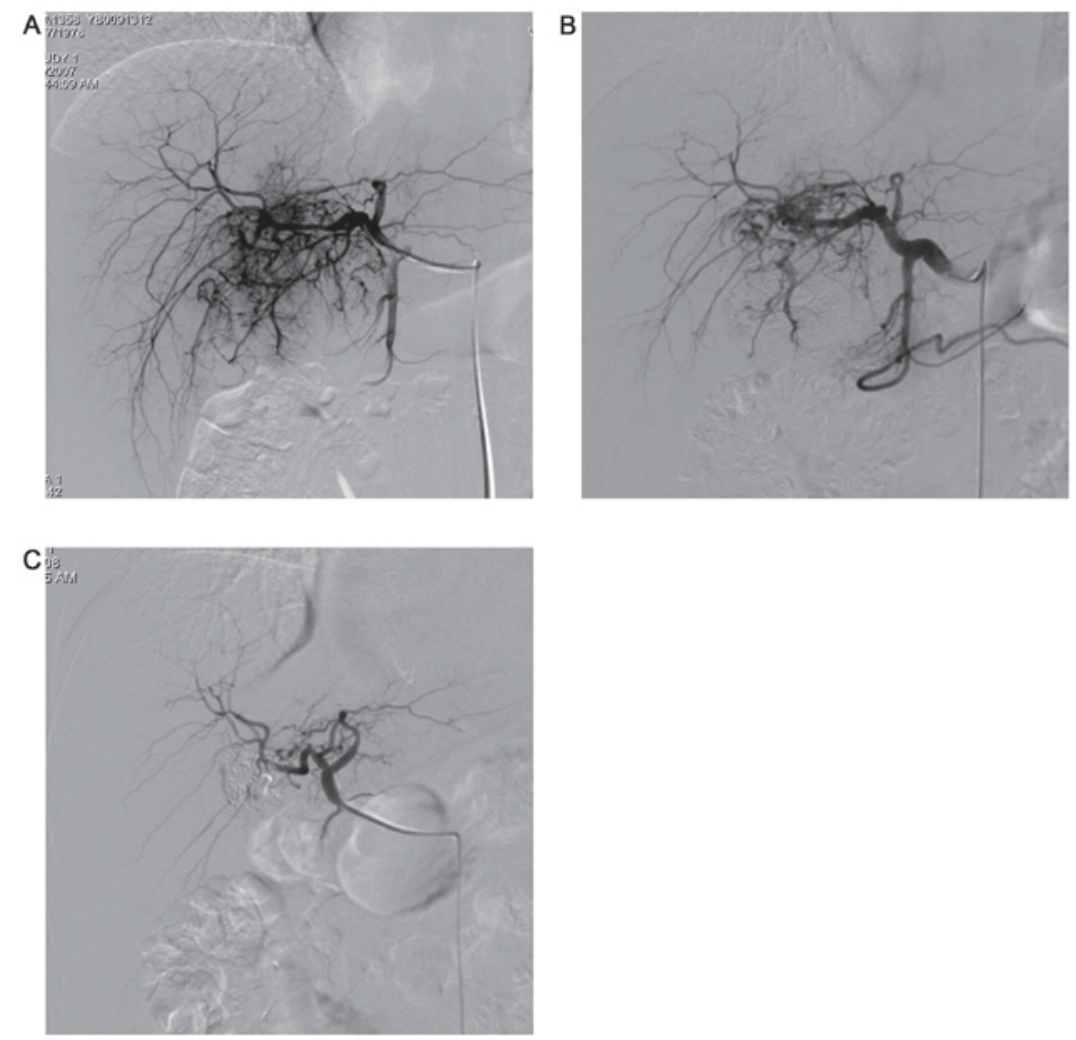

Figure 4. Images from a 30-year-old man with pedunculated hepatocellular carcinoma who received 4 TACE sessions. (A) Angiography prior to TACE demonstrates an extrahepatic tumor growth in the porta hepatis, taking blood from the right hepatic artery, as the main blood supply, and the gastroduodenal artery. (B) Angiographies taken subsequent to 2 sessions of TACE, revealing that the right hepatic artery functions as the main blood supply, whereas the gastroduodenal artery is complementary. (C) Angiographies taken subsequent to 4 sessions of TACE, demonstrating the same phenomenon as observed in (B), with a nearly complete shrinking of the mass. TACE, transcatheter arterial chemoembolization. 
Table II. Angiographic demonstration in 39 patients with pedunculated hepatocellular carcinoma prior to and following TACE.

\begin{tabular}{|c|c|c|c|c|}
\hline Parameter & $\begin{array}{l}\text { Feeding arteries prior to } \\
\text { TACE }^{\mathrm{a}}, \mathrm{n}\end{array}$ & Feeding arteries following repeated $\mathrm{TACE}^{\mathrm{b}}, \mathrm{n}$ & $\chi^{2}$ & P-value \\
\hline Intrahepatic arteries & 39 & 53 (with 14 new intrahepatic collateral vessels) & 4.278 & 0.039 \\
\hline \multicolumn{5}{|l|}{ Extrahepatic collateral arteries } \\
\hline Gastroduodenal artery & 11 & 23 & & \\
\hline Left gastric artery & 6 & 13 & & \\
\hline Inferior phrenic artery & 3 & 11 & & \\
\hline Superior mesenteric artery & 5 & 10 & & \\
\hline Pancreaticoduodenal artery & 4 & 8 & & \\
\hline Right adrenal arteries & 2 & 8 & & \\
\hline Other & 0 & $5^{\mathrm{c}}$ & & \\
\hline
\end{tabular}

${ }^{a} n=70 .{ }^{b} n=131$. Including right gastric arteries $(n=2)$, lumbar arteries $(n=2)$ and an intercostal artery $(n=1)$. TACE, transcatheter arterial chemoembolization.
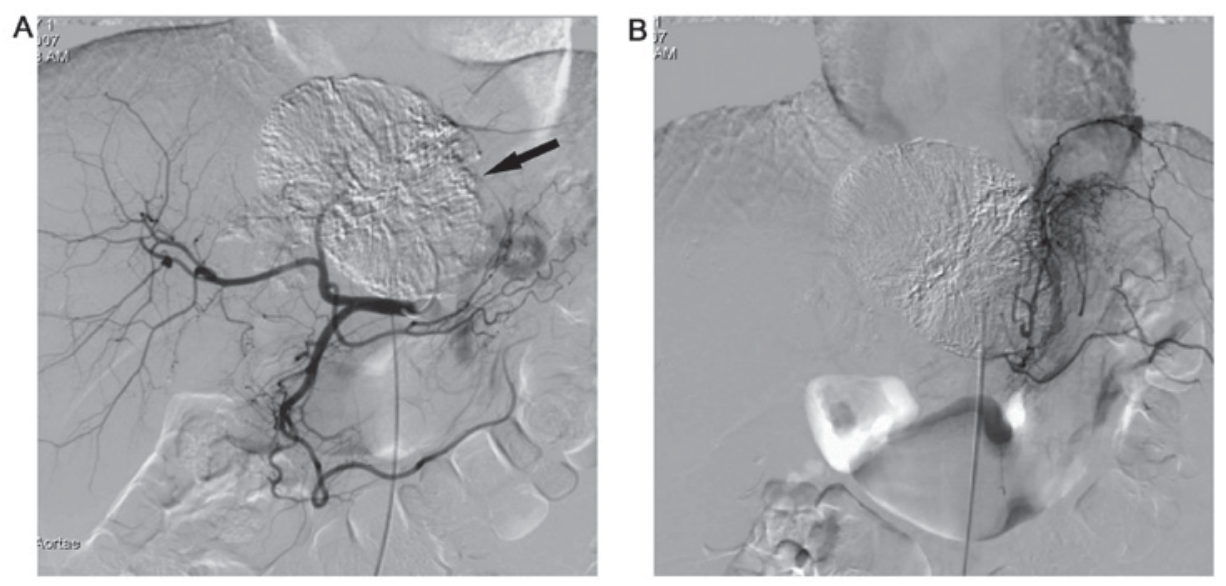

Figure 5. Images from 39-year-old man with an extrahepatic tumor growth on the diaphragmatic surface have underwent 2 sessions of TACE, with slightly high serum $\alpha$-fetoprotein. (A) A hepatic arteriogram performed at the third TACE revealed a small defect in the periphery of the lesion, potentially indicating the presence of active residual tumor tissue (arrow). (B) A catheter is advanced into the left inferior phrenic artery, which adjoins the defect of the lesion and may function as an extrahepatic collateral vessel. The following arteriogram revealed a tumor stain corresponding to the defect presented in the hepatic arteriogram. TACE, transcatheter arterial chemoembolization.

percentage of capsule formation around the tumor, resulting in less vascular invasion than in conventional HCC (5). No symptoms present themselves in patients with early-stage P-HCC; however, once diagnosed, rapid tumor growth ensues, accompanied by intrahepatic metastasis and invasion of neighboring visceral organs (30). Therefore, up to $39.4 \%$ of patients with P-HCC cannot undergo surgical resection (7). If the patients are able to tolerate the procedure, TACE is the first option for the treatment of unresectable HCC, including for patients with intermediate- and advanced-stage disease (8-11,31). The results of the present study demonstrated that the supply of blood to $\mathrm{P}-\mathrm{HCC}$ is complicated and arises from hepatic arteries and extrahepatic collateral supplies (3). Extrahepatic collateral blood supplies may prohibit effective treatment by TACE. For the transcatheter management of P-HCC to be effective, these collateral blood supplies must be adequately embolized (18-28).

TACE treatment has clear limitations for P-HCC tumor control. On one hand, the anatomical features of the extrahepatic collateral vessels, vessels that are often tiny and twisted, make highly selective catheterization and embolization of every feeding artery practically impossible, even when using a microcatheter (23). Additionally, P-HCC tumor lesions adjoin neighboring organs and share feeding arteries (i.e., the origin of extrahepatic collateral supply), which limit arterial injection with chemotherapeutic agents lipiodol emulsion (CALE) and embolization, resulting in poor or no CALE deposition in the tumor (14). Furthermore, a tumor fed by extrahepatic collateral blood supplies may have multiple feeding arteries, as the extrahepatic collateral vessel connects with the hepatic artery and other extrahepatic collateral blood supplies $(12,18)$. Finally, not all extrahepatic collateral supplies are present on the angiographies and so will not receive the chemoembolization. Therefore, TACE alone cannot result in complete tumor necrosis in patients with $\mathrm{P}-\mathrm{HCC}$, and a combination of other therapies, including local ablation and oral sorafenib therapy, should be subsequently utilized (32).

The present study had a number of limitations worth noting. Firstly, there were no patients with conventional HCC enrolled 
as a control for comparison in this retrospective study. Secondly, a microcatheter was not used in highly selective catheterization, particularly in earlier cases, resulting in the omission of a number of extrahepatic collateral supplies. Thirdly, the data only consisted of intermediate or advanced P-HCC, which cannot be treated surgically, unlike early-stage P-HCC. These unresectable cases of P-HCC may form adhesions between the tumor and adjacent organs more readily, which could exaggerate the degree of extrahepatic collateral blood supplies.

The intrahepatic arteries serve as the main blood supply for $\mathrm{P}-\mathrm{HCC}$, whereas the extrahepatic collateral arteries are complementary, regardless of whether patients are pre- or post-TACE. The extrahepatic collateral blood supplies to P-HCC that arises from adjacent vessels are rich, closely associated with tumor size, and are frequently newly established following repeated TACE. As the present study reports treatment at a single institution, the results may not necessarily be applicable to other institutions. The retrospective design and small population size may have resulted in unforeseen bias. Therefore, the results should be validated in a larger prospective study in the future.

\section{Acknowledgements}

This study was supported by the National Natural Science Foundation of China (Grant: 81471730).

\section{References}

1. Eggel H: Uber das primare Carcinom der Leber. Beitr Pathol Anat Allg Pathol 30: 506-604, 1901.

2. Roux: Un cas de cancer primitif du foie avec pericholecystite calculeuse, perforation intestinale: Hemostase hepatique. Rev Med Suisse Romande 17: 114-119, 1897.

3. Horie Y, Katoh S, Yoshida H, Imaoka T, Suou T and Hirayama C: Pedunculated hepatocellular carcinoma. Report of three cases and review of the literature. Cancer 51: 746-751, 1983.

4. Horie Y, Shigoku A, Tanaka H, Tomie Y, Maeda N, Hoshino U, Koda M, Shiota G, Yamamoto T, Kato S, et al: Prognosis for pedunculated hepatocellular carcinoma. Oncology 57: 23-28, 1999.

5. Yeh CN, Lee WC, Jeng LB and Chen MF: Pedunculated hepatocellular carcinoma: Clinicopathologic study of 18 surgically resected cases. World J Surg 26: 1133-1138, 2002.

6. Moritz MW, Shoji M, Sicard GA, Shioda R and DeSchryver K: Surgical therapy in two patients with pedunculated hepatocellular carcinoma. Arch Surg 123: 772-774, 1988.

7. Anthony PP and James K: Pedunculated hepatocellular carcinoma. Is it an entity? Histopatholoy 11: 403-414, 1987.

8. Lopez PM, Villanueva A and Llovet JM: Systematic review: Evidence-based management of hepatocellular carcinoma-an updated analysis of randomized controlled trials. Aliment Pharmacol Ther 23: 1535-1547, 2006.

9. Lo CM, Ngan H, Tso WK, Liu CL, Lam CM, Poon RT, Fan ST and Wong J: Randomized controlled trial of transarterial lipiodol chemoembolization for unresectable hepatocellular carcinoma. Hepatology 35: 1164-1171, 2002.

10. Llovet JM, Real MI, Montana X, Planas R, Coll S, Aponte J, Ayuso C, Sala M, Muchart J, Solà R, et al: Arterial embolisation or chemoembolisation versus symptomatic treatment in patients with unresectable hepatocellular carcinoma: A randomised controlled trial. Lancet 359: 1734-1739, 2002.

11. Yang P, Zeng ZC, Wang BL, Zhang JY, Fan J, Zhou J and Hu Y: The degree of Lipiodol accumulation can be an indicator of successful treatment for unresectable hepatocellular carcinoma (HCC) patients-in the case of transcatheter arterial chemoembolization (TACE) and external beam radiotherapy (EBRT). J Cancer 7: 1413-1420, 2016.

12. Miyayama S, Matsui O, Taki K, Minami T, Ryu Y, Ito C, Nakamura K, Inoue D, Notsumata K, Toya D, et al: Extrahepatic blood supply to hepatocellular carcinoma: Angiographic demonstration and transcatheter arterial chemoembolization. Cardiovasc Intervent Radiol 29: 39-48, 2006.
13. Yuminaga Y, Kam J and Louie-Johnsun M: Multi-centre, prospective evaluation of the Seldinger technique for difficult male urethral catheter insertions by non-urology trained doctors. BJU Int 120: (Suppl 3): S21-S27, 2017.

14. Huang D, Chen Y, Chen S, Zeng Q, Zhao J, Wu R and Li Y: TACE plus percutaneous chemotherapy-lipiodol treatment of unresectable pedunculated hepatocellular carcinoma. Medicine 96: e7650, 2017.

15. Charnsangavej C, Chuang VP, Wallace S, Soo CS and Bowers T: Angiographic classification of hepatic arterial collaterals. Radiology 144: 485-494, 1982.

16. Michels NA: Collateral arterial pathways to the liver after ligation of the hepatic artery and removal of the celiac axis. Cancer 6 : 708-724, 1953.

17. Koehler RE, Korobkin M and Lewis F: Arteriographic demonstration of collateral arterial supply to the liver after hepatic artery ligation. Radiology 117: 49-54, 1975.

18. Miyayama S, Matsui O, Akakura Y, Yamamoto T, Nishida H, Yoneda K, Kawai K and Nishijima H: Hepatocellular carcinoma with blood supply from omental branches: Treatment with transcatheter arterial embolization. J Vasc Interv Radiol 12: 1285-1290, 2001.

19. Chung JW, Park JH, Han JK, Choi BI, Kim TK and Han MC: Transcatheter oily chemoembolization of the inferior phrenic artery in hepatocellular carcinoma: The safety and potential therapeutic role. J Vasc Interv Radiol 9: 495-500, 1998.

20. Hirota S, Matsumoto S, Fukuda T, Yoshikawa T, Motohara T and Ichikawa S: Solitary hepatocellular carcinoma fed by the cystic artery: Limitation of transcatheter arterial embolization. Cardiovasc Intervent Radiol 22: 206-209, 1999.

21. Tanigawa N, Sawada S, Okuda Y, Shinzato S, Mishima K, Asai T, Ohmura N and Kobayashi M: A case of small hepatocellular carcinoma supplied by the cystic artery. AJR Am J Roentgenol 170: 675-676, 1998.

22. Park SI, Lee DY, Won JY and Lee JT: Extrahepatic collateral supply of hepatocellular carcinoma by the intercostal arteries. J Vasc Interv Radiol 14: 461-468, 2003.

23. Soo CS, Chuang VP, Wallace $\mathrm{S}$, Charnsangavej $\mathrm{C}$ and Carrasco $\mathrm{H}$ : Treatment of hepatic neoplasm through extrahepatic collaterals. Radiology 147: 45-49, 1983

24. Kim JH, Chung JW, Han JK, Park JH, Choi BI and Han MC: Transcatheter arterial embolization of the internal mammary artery in hepatocellular carcinoma. J Vasc Interv Radiol 6: 71-77, 1995.

25. Duprat G, Charnsangavej $\mathrm{C}$, Wallace $\mathrm{S}$ and Carrasco $\mathrm{CH}$ : Inferior phrenic artery embolization in the treatment of hepatic neoplasms. Acta Radiol 29: 427-429, 1988.

26. Nakai M, Sato M, Kawai N, Minamiguchi H, Masuda M, Tanihata H, Takeuchi T, Terada M and Kishi K: Hepatocellular carcinoma: Involvement of the internal mammary artery. Radiology 219: 147-152, 2001.

27. Kodama Y, Shimizu T, Endo H, Hige S, Kamishima T, Holland GA, Miyamoto N and Miyasaka K: Spontaneous rupture of hepatocellular carcinoma supplied by the right renal capsular artery treated by transcatheter arterial embolization. Cardiovasc Intervent Radiol 25: 137-140, 2002.

28. Miyayama S, Matsui O, Nishida H, Yamamori S, Minami T, Shinmura R, Kozaka K, Notsumata K, Toya D, Tanaka N, et al: Transcatheter arterial chemoembolization for unresectable hepatocellular carcinoma fed by the cystic artery. J Vasc Interv Radiol 14: 1155-1161, 2003.

29. Miyayama S, Matsui O, Taki K, Minami T, Ito C, Shinmura R, Takamatsu S, Kobayashi M, Notsumata K, Toya D, et al: Transcatheter arterial chemoembolization for hepatocellular carcinoma fed by the reconstructed inferior phrenic artery: Anatomical and technical analysis. J Vasc Interv Radiol 15: 815-823, 2004.

30. Nishizaki T, Matsumata T, Adachi E, Hayashi $\mathrm{H}$ and Sugimachi K: Pedunculated hepatocellular carcinoma and surgical treatment. Br J Cancer 67: 115-118, 1993.

31. Chung GE, Lee JH, Kim HY, Hwang SY, Kim JS, Chung JW, Yoon JH, Lee HS and Kim YJ: Transarterial chemoembolization can be safely performed in patients with hepatocellular carcinoma invading the main portal vein and may improve the overall survival. Radiology 258: 627-634, 2011.

32. Becher G, Sozgen T, Olschewski M, Laubenberger J, Blum HE and Allgaier HP: Combined TACE and PEI for paliative treatment of unresectable hepatocellular carcinoma. World J Gastroenterol 11: 6104-6109, 2005. 